\title{
MODELLING AND SIMULATION OF THE DYNAMIC CUTTING PROCESS AND SURFACE TOPOGRAPHY GENERATION IN NANO/MICRO CUTTING
}

\author{
Lei Zhou ${ }^{1,2}$, Kai Cheng ${ }^{1}$
}

1. School of Engineering and Design, Brunel University, Uxbridge, UK, UB8 3PH

2. School of Mechatronics Engineering, Harbin Institute of Technology, Harbin, China, 150001

\section{Abstract}

In nano/micro cutting process, the surface quality is heavily dependent on all the dynamic factors in machining including those from the material, tooling, cutting parameters, servo accuracy, mechanical structure deformation, and non-linear factors as well. The machined surfaces are generated based on the tool profile and the real tool path combining with the various external and internal disturbances. To bridge the gap between the machining conditions and the surface quality, the integrated simulation system presented involves the dynamic cutting process, control/drive system and surface generation module. It takes account all the intricate aspects of the cutting process, such as material heterogeneity, regenerative chatter, built-up edge (BUE), spindle run-out, environmental vibration, and tool interference, etc. The frequency ratio method is used to interpret the surface topography and texture formation. The proposed systematic modelling approach is verified by the cutting experiment.

Keywords: Dynamic cutting force, non-linear factors, surface topography, tool interference.

\subsection{Introduction}

Metal cutting, especially in nano/micro scale, is a complex process which comprises workpiece material, tooling geometry, cutting parameters, servo drive accuracy, static and dynamic deformations of structure in machining, etc. The machined surfaces are generated based on the tool profile and real tool path consider with various disturbances. It is of great benefit to build a systematic model and analyze the effects from the dynamic cutting process. Many linear/nonlinear factors in the whole system will contribute to the surface quality. The dynamic cutting force joints all the factors together, and produce the tool-workpiece relative displacement in the stiffness loop. 
Take 3-Axis turning machine as a case in point, shown as Fig. 1, the stiffness loop in face cutting process involves: spindle axial runout, workpiece clamp error, workpiece material property, cutting tool geometry, tool holder stiffness, $\mathrm{Z}$ axis servo stiffness in axial direction, $\mathrm{X}$ axis slideway stiffness in side direction, and mounting stiffness. In this stiffness loop, all the elements are guaranteed by physical construction like bearings, mounting screws and other mechanical structures except the linear motor in axial direction. Since employ the direct driven linear motor, the stiffness in $\mathrm{Z}$ axial motion is directly provided by electrical servo motor. The tuning of $\mathrm{Z}$ axis linear motor, in the error sensitive direction, is one of the most critical ingredients of ultra-precision machine tool.

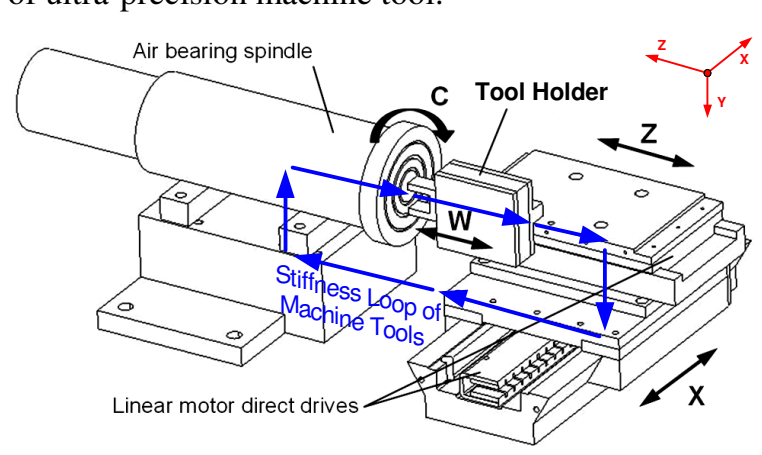

Fig. 1 Stiffness Loop of 3-Axis Turning Machine

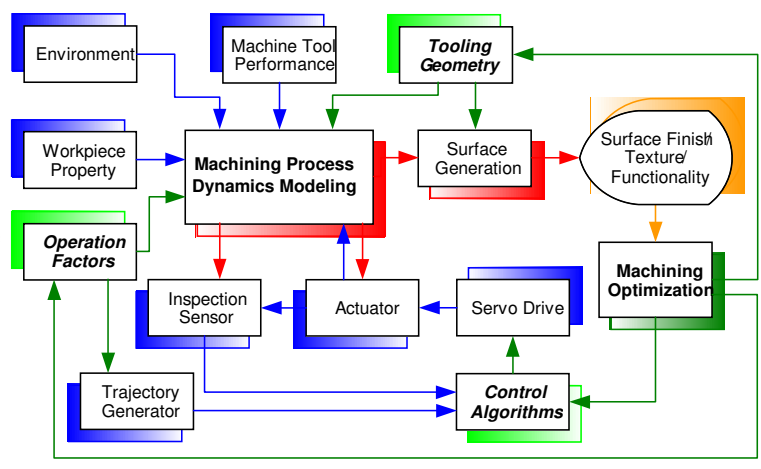

Fig. 2 Machining Process Integrated System

To get the connection of the tool path and the machined surface, several literatures focuses the surface topography generation process. Cheung and Lee ${ }^{[1]-[2]}$ used the digitizing oscilloscope to record the relative displacement between the tool and the workpiece. After been analyzed by Fast Fourier Transform (FFT), the main frequency and amplitude of vibration reconstructed the tool deviation from ideal position. The tool profile superimposed onto the tool path to generate the surface topography. Lee and Cheung ${ }^{[3]-[4]}$ built a dynamic cutting system to predict the surface topography in face turning. However the cutting force model only contained the linear factors and the whole cutting system was simplified into a second-order system. The servo control system, material hard grain effect, chatter ${ }^{[5]}$, tool wear, spindle runout and environmental vibration were not taken into account. Luo et al. ${ }^{[6]-[7]}$ introduced some non-linear factors to the cutting process and studied the effects of machining process variables and tooling characterizations on the surface texture, yet the tool interference effect in nano/micro scale cutting process had not been involved.

In short, for micro/nano cutting process, the machined surface performance is all about how successfully the tool-workpiece relative position meets its desired objective. The topography and texture of cutting surface will be generated by the dynamic feed response under the multifarious cutting factors.

In this paper, the dynamic cutting process system is established. The machined surface topography is predicted though the real tool path and tooling geometry. From analyzing the cutting force, the tool-workpiece relative displacement and the surface profile, the origins of surface error are located to the dynamic cutting factors. The preliminary cutting trials certify the validity of the dynamic cutting system modelling.

\subsection{Systematic Modelling of the Dynamic Nano/micro Cutting}

The generation of workpiece surface is a very complex material removal process as shown in Fig.2 including:

- servo control dynamics, which is joined by control algorithm, servo amplifiers, actuators and inspection sensors;

\section{MODELLING AND SIMULATION OF THE DYNAMIC CUTTING PROCESS AND SURFACE TOPOGRAPHY GENERATION IN NANO/MICRO CUTTING}


- machining process dynamics, which is highly affected by six main aspects: machine tool performance, cutting tool geometry, workpiece material properties, operation factors, servo motion and environment; and

- $\quad$ surface generation and surface texture, integrity and functionality analysis.

To get a better understanding of the complex process, a systematic model is developed in the MATLAB Simulink module as Fig. 3. The modelling approach bridges the gap between the determinative factors and the surface generation. Based on a thorough theoretical analysis of servo motions and cutting mechanics/dynamics, the integrated model produces a scientific methodology to simulate the precision surface generation in nano/micro turning processes.

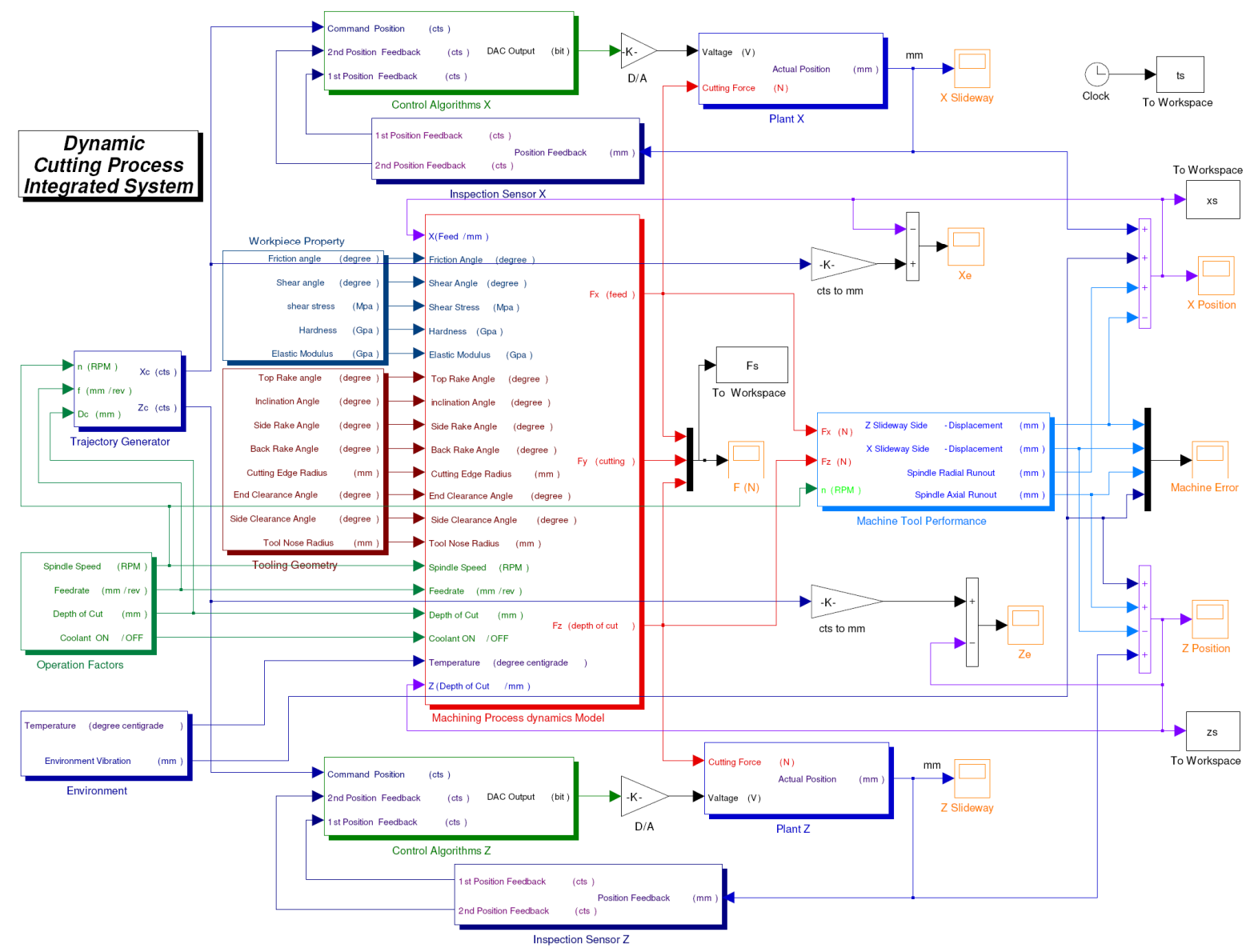

Fig. 3 Dynamic Machining Process Integrated Modelling

\subsection{Modelling of Servo Control Dynamics}

Most of the cutting dynamics simulations are simplified model the control and drive system as a second-order system. However, the advanced control algorithms are always applied on the ultra precision machine tools to achieve higher servo accuracy. Modelling the control system can also help to modify the controller construction and finally improve the surface quality. In this paper, the ultra precision turning machine employs the UMAC motion controller who provides a PID position/velocity servo control scheme with velocity and 
acceleration feedforward loops ${ }^{[8]}$. The discrete model uses Z-transforms whose sampling period is the real servo-interrupt cycle. The position quantification based on the encoder resolution is $4.88 \mathrm{~nm}$.

The output from the UMAC controller is lead to servo amplifier to continue the current loop. The direct-drive linear motor model is built on the resistance and inductance of the motor windings, force constant and back EMF constant from the specifications. In this case, the static air bearing slideway is applied to reduce the friction and unexpected load in the motion direction. Therefore, the plant of the slide carriage is simplified represent as a pure mass module.

From servo control dynamics Model, the actual motion of each slideway is calculated for the next stage to generate the real tool path. In fact, there are some additional disturbances in the drive system: electrical noise in the current loop and cutting force disturbance superimposed on the sideways which is generated by dynamic cutting process model.

\subsection{Dynamic Cutting Process}

The quality of the machined surface is mainly determined by the relative motions between the cutting tool edge and the workpiece. In practice, the tool path deviates from the ideal tool path as a result of kinematic or dynamic factors from the dynamic cutting process, machine tool motion errors and environmental disturbances. For instance, the deformation of the slideway bearings under the action of dynamic cutting forces will deflect the cutting tool nose point; environmental vibrations will make the motion of the cutting tool away from the designed tool path.

Since the surface topography model basically follows the reflection of cutting tool edge profile, to calculate the real tool path becomes the critical part in the modelling approach. Table I lists the linear and nonlinear factors from the structural deformation and motion errors of the machine tool and cutting tool which will contribute the deviation of the tool path.

Table I: Linear and Nonlinear Factors in the Cutting Process

\begin{tabular}{|c|c|c|c|}
\hline Source & Influence Factor & \multicolumn{2}{|c|}{ Mathematical Function } \\
\hline BUE & Rake Angle & $\Delta \alpha=\alpha_{A} \operatorname{Rse}\left(\omega_{\alpha} t\right)$ & (1) \\
\hline Hard Grain & Shear Stress & $\Delta \tau_{l}=\tau_{A} \operatorname{Pul}(t)$ & $(2)$ \\
\hline Coolant & Friction Angle & $\Delta u=r(t)$ & (3) \\
\hline Flank Wear & Tool Wear & $A F_{r} y+B \exp (-E)^{[9-10]}$ & (4) \\
\hline $\begin{array}{c}\text { Regenerative } \\
\text { Vibration }\end{array}$ & $\begin{array}{c}\text { Feed and Depth of } \\
\text { Cut Chatter }\end{array}$ & $\begin{array}{l}\Delta c_{t}=Z(t)-Z(t-T) \\
\Delta c_{w}=X(t)-X(t-T)\end{array}$ & (5) \\
\hline Spindle Runout & \multirow{3}{*}{$\begin{array}{c}\text { Displacement } \\
\text { between Cutting } \\
\text { Tool and Workpiece }\end{array}$} & $E_{s}=A_{s} \sin (\omega t+\phi)$ & $(6)$ \\
\hline Slideway Stiffness & & $e_{s}=F_{s} / k_{s}$ & (7) \\
\hline $\begin{array}{c}\text { Environmental } \\
\text { Vibration }\end{array}$ & & $E_{v}=A_{e v} \sin \left(2 \pi f_{e v} t\right)$ & (8) \\
\hline
\end{tabular}

where $\Delta \alpha$ is the variation of the rake angle due to BUE, $\alpha_{A}$ and $\omega_{\alpha}$ are the amplitude and frequency of the variation of the rake angle due to BUE, Rse is a function to generate an arbitrarily shaped period signal; $\Delta \tau_{l}$ is the increment of the shear stress due to the hard grain, $\tau_{A}$ is the amplitude of the increment of the shear stress due to the hard grain; Pul is the Pulse function to generate square wave; $\Delta u$ is the change of the friction angle between the tool rake face and chip, $r$ is the step function; $l_{w}$ is the tool flank wear width, $F_{r}$ is the resultant

\section{MODELLING AND SIMULATION OF THE DYNAMIC CUTTING PROCESS AND SURFACE TOPOGRAPHY GENERATION IN NANO/MICRO CUTTING}


cutting force, $H_{t}$ is the hardness of the cutting tool material, $V$ is the cutting speed, $V_{s}$ is the sliding speed, $E$ is the process activation energy, $R$ is the universal gas constant, $T_{f}$ is the cutting temperature in the tool flank zone, $A$ and $B$ are the constants; $\Delta c_{t}$ is the variation of cutting thickness, $\Delta c_{w}$ is the variation of cutting width; $E_{s}$ is the spindle synchronous error, $A_{s}, \omega$ and $\phi$ is the amplitude, spindle angular speed and phase shift of the spindle axial runout; $e_{s}$ is the error of the slideway in $\mathrm{X} / \mathrm{Y} / \mathrm{Z}$ direction, $k_{s}$ is the stiffness of the slideway in $\mathrm{X} / \mathrm{Y} / \mathrm{Z}$ direction, $F_{s}$ is the cutting forced in $\mathrm{X} / \mathrm{Y} / \mathrm{Z}$ direction; $E_{v}$ is the environmental vibration, $f_{e v}$ and $A_{e v}$ are the frequency and amplitude of the environmental vibration.

The demonstrated workpiece material is the Aluminum alloy, thus the dynamic cutting force model will follow the elastic-plastic mechanics model. Based on the elastic-plastic deformation principle, the forces acting on the rake face can be acquired by the coordinate transformation of the shear plane force based on the shear plane cutting model. The force acting on the cutting edge and flank face can be deduced based on the empirical formula of the contact stress and elastic recovery. Accumulating the forces action on the three zones, there will be the dynamic cutting forces in the three directions following the Cartesian coordinate system in Fig. 1 expressed as ${ }^{[11]}$ :

$$
\left\{\begin{aligned}
F_{x}(t) & =\left(K_{f c} \sin \theta_{r}+1-\cos \theta_{r}\right)\left[d_{c}+z(t)-z(t-T)\right]\left\{[h+x(t)-x(t-T)]+\sqrt{R_{0}^{2}-\left[R_{0}-d_{c}-z(t)+z(t-T)\right]^{2}}\right\} \\
& +\left(K_{f e} \sin \theta_{r}+1-\cos \theta_{r}\right) R_{0} \theta_{r}+K_{f f 1} \cdot\left(\cos \frac{\beta}{2}+\mu \sin \frac{\beta}{2}\right)+K_{f f 2} \cdot(\cos \beta+\mu \sin \beta) \\
F_{y}(t) & =K_{t c} \cdot\left[d_{c}+z(t)-z(t-T)\right]\left\{[h+x(t)-x(t-T)]+\sqrt{R_{0}^{2}-\left[R_{0}-d_{c}-z(t)+z(t-T)\right]^{2}}\right\} \\
& +K_{t e} R_{0} \theta_{r}+K_{t f 1} \cdot\left(\sin \frac{\beta}{2}-\mu \cos \frac{\beta}{2}\right)+K_{t f 2} \cdot(\sin \beta-\mu \cos \beta) \\
F_{z}(t) & =\left(K_{f c}-K_{r c}\right) \sin \theta_{r}\left[d_{c}+z(t)-z(t-T)\right]\left\{[h+x(t)-x(t-T)]+\sqrt{R_{0}^{2}-\left[R_{0}-d_{c}-z(t)+z(t-T)\right]^{2}}\right\}+K_{t e} R_{0} \theta_{r} \sin \theta_{r}
\end{aligned}\right.
$$

where $h$ is the undeformed chip thickness, $f$ is the feed rate, $\mu$ is the friction angle coefficient, $R_{0}$ is the tool nose radius and $d_{c}$ is the depth of cut, $\beta$ is the side clearance angle, $\theta_{r}$ is the intersection angle of two continuous tool paths, $T$ is the spindle revolution period, $K_{t c}, K_{r c}, K_{f c}, K_{t e}, K_{r e}, K_{f e}, K_{t f l}, K_{t f 2}, K_{f f f l}$ and $K_{f f 2}$ are the cutting constants at rake face, cutting edge and flank face in $X, Y$ and $Z$ directions, which can be acquired by the transformation from the orthogonal cutting experiments and an empirical tool force model.

As has been stated, the real tool path $\left(X_{t p}, Z_{t p}\right)$ is created by all the above factors: servo motions influenced by dynamic cutting disturbance $\left(X_{s m}, Z_{s m}\right)$, machine tool deformations (spindle radial runout $E_{s r}$, spindle axial runout $E_{s a}, \mathrm{Z}$ slideway side-stiffness $K_{z x}, \mathrm{X}$ slideway side-stiffness $\left.K_{x z}\right)$ and environmental vibrations $\left(E_{e v x}\right.$, $E_{\text {evz }}$ ) shown as:

$$
\left\{\begin{array}{l}
X_{t p}=X_{s m}+E_{e v x}+E_{s r}-F_{x} / K_{z x} \\
Z_{t p}=Z_{s m}+E_{e v z}+E_{s a}-F_{z} / K_{x z}
\end{array}\right.
$$

\subsection{Simulation of Nano/micro Machined Surfaces}

\subsection{Prediction Algorithms for a Machined Surface}

The last stage of machining system is to renders the surface topography. The cutting tool will follow the real tool path to reproduce the tool profile on the machined surface in the form of feed marks.

MODELLING AND SIMULATION OF THE DYNAMIC CUTTING PROCESS AND SURFACE TOPOGRAPHY GENERATION IN NANO/MICRO CUTTING 
Cheung et al. ${ }^{[12]}$ and Kim et al. ${ }^{[13]}$ calculate the intersection of two adjacent tool profiles to get the boundary of each feed marks like Fig.8 (a). However, in the ultra-precision machining, whose feedrate is so small that the next several cuts will clean up the previous tool marks. As shown in Fig.4 (b), the $(i+1)$ th and $(i+2)$ th tool profiles which are over cut by the $(i+3)$ th can not affect the workpiece surface generation. The machined surface is contoured by the $i$ th, $(i+3)$ th, $(i+4)$ th, etc. tool profiles as a result of tool interference from vibrations. Frankly speaking, tool path vibration is not the entire reason to form the surface topography in this case. The tool interference is normal in the small feedrate cutting process, and it always help to flat the toolworkpiece vibration in this case.

All the 3D surface topography prediction procedures are described in Fig. 5 as a block flow diagram. The principal advantage of this approach is considering the multiple tool interference which is more coincident with the real practice.

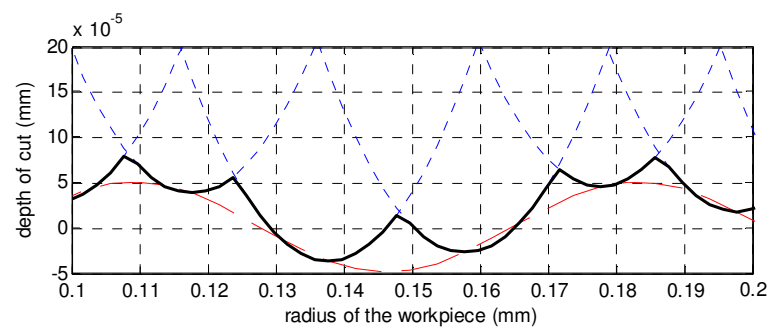

(a) Intersections of two adjacent tool profiles

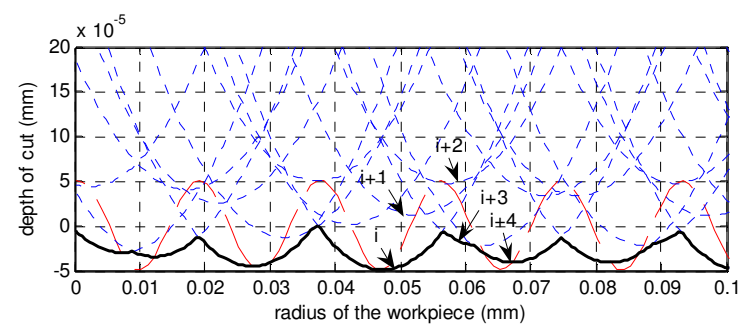

(b) Intersections in consideration of all tool profiles

Fig. 4 2D Machined Surface Profile simulation

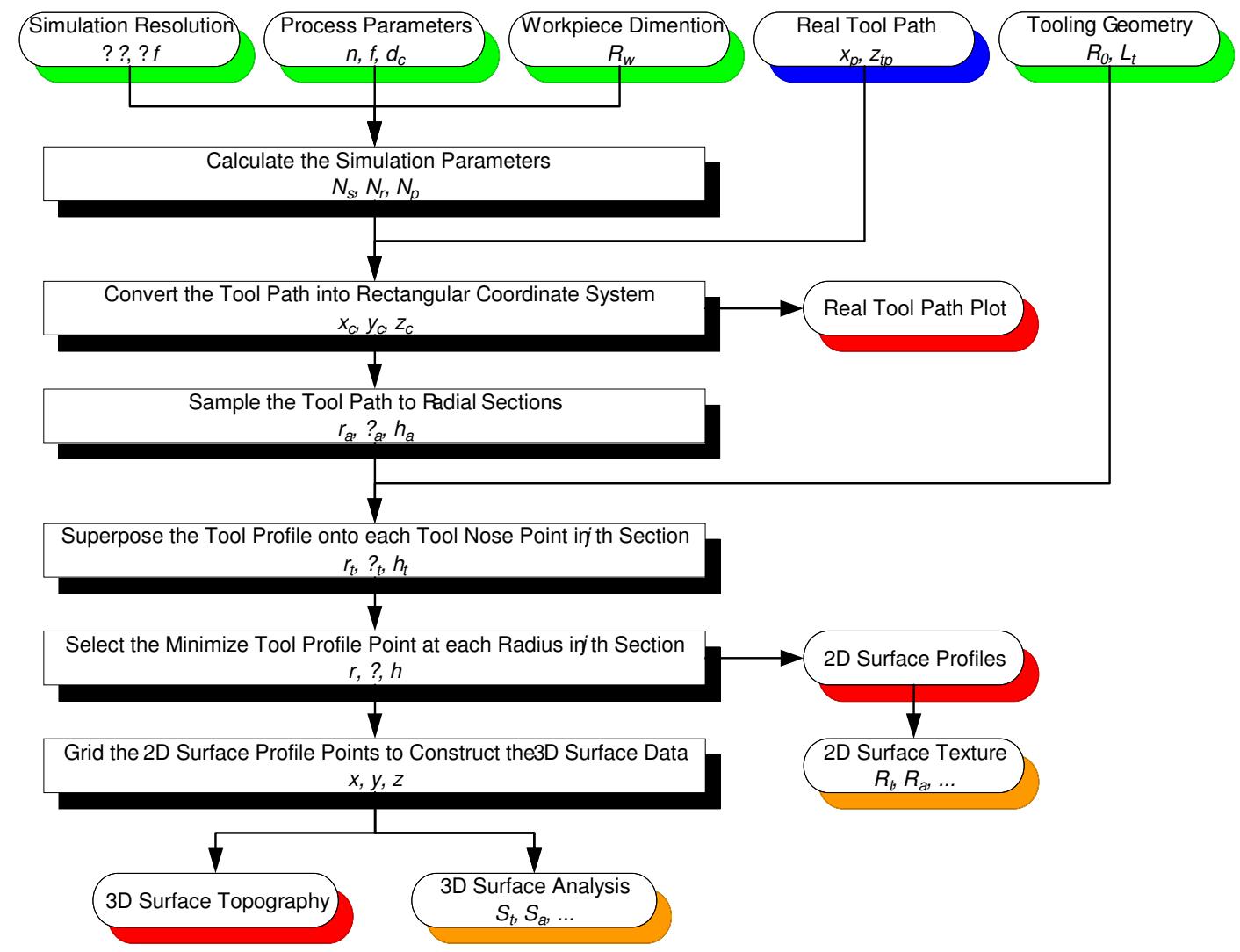

Fig. 5 The Flow Chart of 3D Surface Topography Simulation Model

MODELLING AND SIMULATION OF THE DYNAMIC CUTTING PROCESS AND SURFACE TOPOGRAPHY GENERATION IN NANO/MICRO CUTTING 


\subsection{Simulation on the Surface Generation}

The relative displacement between the tool and the workpiece in $\mathrm{Z}$ direction is mainly decided by workpiece material, machine tool performance, tooling geometry and cutting parameters. The simulated surface under the dynamic cutting process, cut from edge to centre, is a $1.4 \mathrm{~mm} \times 1.4 \mathrm{~mm}$ square located at the spindle central line as Fig. 6.

The impressive features on the surface are the rings and flutes which are much bigger than the tool feed mark size. To analysis the waviness structure regulation, it is better to work out the connection of the vibrations and the machined surface topography. Since the tool paths in one section are the discrete points by feed spacing per spindle revolution, the surface generation is like the process of cutting tool edge in feed direction sampling the vibratory tool path. The machined surface topography under vibration is directly decided by the relation between the vibration frequency $f_{v}$ and the spindle rotational frequency $f_{s}$. Define the frequency ratio $f_{r}$ as:

$$
f_{r}=\frac{f_{v}}{f_{s}}=\frac{f_{v}}{n / 60}=a+b
$$

where $a$ is the integer part of the ratio and $b$ is the fractional part in the rage of -0.5 to 0.5 . In this case namely, $f_{r}$ is the frequency that the tool tip traverses the vibration within one spindle rotation period. The number of the flutes is identical to the integer part $a$, which means how many times the vibration is completely undergone per spindle revolution. The absolute value $|b|$, which can not cover the full vibration cycle, will make the phase offset in each spindle rotation. The orientation of the flutes is decided by the sign of $b$. Positive sign means counter-clockwise $(\mathrm{CCW})$; negative sign is clockwise $(\mathrm{CW})$. In other words, the radial section presents the fractional part of the frequency ratio $b$, while the integer part $a$ will be reflected in the circumferential direction.

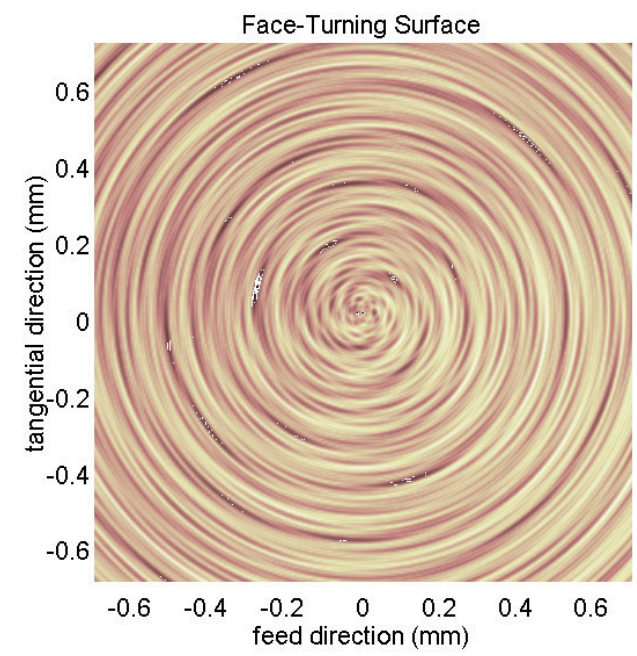

Fig. 6 Flat Turning Surface Simulation

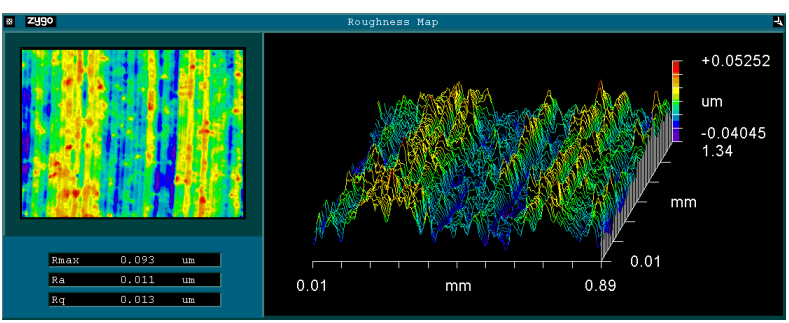

(a) Measurement of the Actual Machined Surface

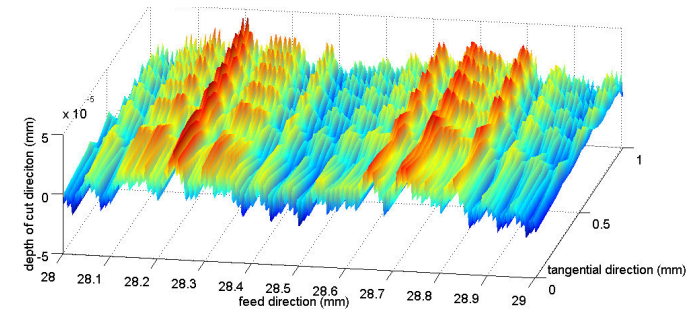

(b) Simulation of the Machined Surface

Fig.7 Identification of the Surface Prediction System 
The $6^{\text {th }}$ International Conference on Manufacturing Research (ICMR08)

Brunel University, UK, 9-1 ${ }^{\text {th }}$ September 2008

\subsection{Experimental Verification}

In order to evaluate the effectiveness of the dynamic cutting process model and the surface prediction method, the surface descript so far were verified through the experimental work. Fig.7 (a) shows the measurement result of an actual Al specimen machined at $1000 \mathrm{RPM}$ spindle speed, $0.01 \mathrm{~mm} / \mathrm{rev}$ feedrate, $0.01 \mathrm{~mm}$ depth of cut, and $0.508 \mathrm{~mm}$ tool nose radius using Zygo NV 5000 system. The measured surface locates on the 28$29 \mathrm{~mm}$ radius area where the tool marks are nearly the straight lines. The surface prediction is simulated as Fig.7 (b) whose form is accordant with the experiment result.

\subsection{Conclusion}

- Metal cutting process is combined with the material property, cutting chatter, tooling geometry, servo capability, mechanical performance, and environmental vibration, etc. The integrated simulation system can help to better understand the connection of the dynamic cutting process and the surface generation. The non-linear factors will simulate the real cutting condition, such as hard grain of material, BUE, regenerative vibration, tool wear and spindle runout. Building the drive and control system in the model gives the flexibility to evaluate and improve the control system performance.

- Surface prediction should take consider of tool interference effect. The tool interference commonly appears when the low feedrate or big rake radius cutting tool is adopted. The valleys on the machined surface may be not caused by the tool interference, but from the tool path vibration.

- The frequency ratio method can interpret the surface topography formation. In this case, $a$ states the number of the flutes; $|b|$ is the phase offset in one section and the sign of $b$ present the flutes direction.

- The computer simulation and preliminary experimental results have proved that the approach is able to identify any existence of tool vibrations in nano/micro cutting process and their effect on the surface generation. Currently, the authors are undertaking well-designed substantial cutting trials and simulations to further verify the approach developed potentially applied to free-form surfaces in multi-axis nano/micro machining, the results will be presented in other papers in the near future.

\section{Acknowledgement}

The authors wish to acknowledge the assistance and support of the EU sixth Framework IP MASMICRO project (contract NMP2-CT-2004-500095-2). In particular we wish to thank our partners in the RTD 5 subgroup.

\section{References}

[1] C.F. Cheung, W.B. Lee. Characterisation of nanosurface generation in single-point diamond turning. International Journal of Machine Tools \& Manufacture. 2001, 41, 851 875

[2] C.F. Cheung, W.B. Lee. A theoretical and experimental investigation of surface roughness formation in ultraprecision diamond turning. International Journal of Machine Tools \& Manufacture. 2000, 40, 979 1002

[3] W.B. Lee, C.F. Cheung. A dynamic surface topography model for the prediction of nano-surface generation in ultraprecision machining. International Journal of Mechanical Sciences, 2001, 43, 961 991

[4] W.B. Lee, C.F. Cheung, etc. Develop of a virtual machining and inspection system for ultra-precision diamond turning. Proc. IMechE. Part B: Journal of Engineering Manufacture, 2007, 221, 1153 1174

[5] A.A. Cardi, et al. Workpiece dynamic analysis and prediction during chatter of turning process, Mechanical Systems and Signal Processing, 2007

[6] X. Luo, K. Cheng and R. Ward. The effects of machining process variables and tooling characterizations on the surface generation, International Journal of Advanced Manufacturing Technology. 2005, 25, 1089 1097 
The $6^{\text {th }}$ International Conference on Manufacturing Research (ICMR08)

Brunel University, UK, 9-11 ${ }^{\text {th }}$ September 2008

[7] X. Luo and K. Cheng. Nonlinear effects in precision machining of engineering materials, American Society of Precision Engineering Annual Meeting, 2003, 489 493

[8] Delta Tau Data Systems, Inc, Turbo PMAC User Manual, 2006

[9] T.H.C. Childs, K. Maekawa, T. Obikawa and Y. Yamane. Metal Cutting: Theory and Applications, Arnold, London, 2000

[10] C. Schmidt, P. Frank, H. Weule, J. Schmidt, Y.C. Yen and T. Altan. Tool wear prediction and verification in orthogonal cutting. 6th CIRP International Workshop on Modeling of Machining Operations. Hamilton, Canada, May 20, 2003

[11] Yusuf Altintas. Manufacturing automation: metal cutting mechanics, machine tool vibrations, and CNC design. Cambridge University Press, Cambridge, 2000

[12] C.F. Cheung, W.B. Lee. Modelling and simulation of surface topography in ultra-precision diamond turning. Proc. IMechE. Part B: Journal of Engineering Manufacture, 2000, 214, 463 480

[13] Dong-Sik Kim, In-Cheol Chang, Seung-Woo Kim. Microscopic topographical analysis of tool vibration effects on diamond turned optical surfaces, Precision Engineering. 2002, 26, 168 174 